\title{
CONTINUOUS FUNCTION SPACES ISOMETRIC TO HILBERT SPACE ${ }^{1}$
}

WILLIAM F. DONOGHUE, JR.

A well-known theorem due to Banach [2, p. 187] states that every separable (real) Banach space is isometric to a subspace of $C(0,1)$, the continuous real functions on the unit interval with norm $\|x\|$ $=\sup _{t}|x(t)|$. It is natural to ask for those subspaces of $C(0,1)$ which are isometric to finite or infinte dimensional Hilbert space. Although such questions are properly considered in the light of the general theory of reproducing kernels [1], that theory will not be explicitly invoked in this note which is intended only to show that subspaces of $C(0,1)$ isometric to Hilbert space consist of functions of very pathological type. All spaces considered in this note are real, and $R_{k}$ is the $k$-dimensional real Euclidean space.

TheOREM. If $\mathcal{H C}$ is a subspace of $C(0,1)$ isometric to Hilbert space; if $N=\operatorname{dim}\left(\right.$ FC) (which may be infinite) and $x_{i}(t), i=1,2, \cdots, k$, are $k$ linearly independent elements of $\mathfrak{H}$, where $k<N$, then the curve in $R_{k}$ described by the point $X(t)=\left(x_{i}(t)\right)$ is a space-filling curve there, i.e. covers a sphere in $R_{k}$.

Proof. The functional $\psi_{t}$ on $\mathcal{H}$ which is the evaluation functional at the point $t, \psi_{t}(x)=x(t)$ is clearly continuous and linear and therefore corresponds to an inner product: $\psi_{t}(x)=x(t)=\left(x, K_{t}\right)$ with $K_{t}$ in FC. Accordingly there exists a mapping of the unit interval onto a subset $K$ of $\mathcal{H}$ which is continuous in the weak topology of $\mathcal{H}$, since for all $x$ the function $\left(x, K_{t}\right)$ is continuous in $t$. Thus $K$ is weakly compact. Moreover, $K$ is contained in the unit sphere of $\mathfrak{F}$ :

$$
\left\|K_{s}\right\|=\sup _{s}\left|K_{s}(t)\right| \geqq\left|K_{s}(s)\right|=\left(K_{s}, K_{s}\right)=\left\|K_{s}\right\|^{2}
$$

whence $\left\|K_{\triangleleft}\right\| \leqq 1$.

Let $x$ be an arbitrary element of unit norm in $\mathfrak{F}$; then $\|x\|$ $=\sup _{t}|x(t)|=\sup _{t}\left|\left(x, K_{t}\right)\right|$ and the supremum is attained at some point $s$ in the interval. Thus by the Schwartz inequality $\|x\|=|x(s)|$ $=\left|\left(x, K_{8}\right)\right| \leqq\|x\|\left\|K_{s}\right\| \leqq\|x\|$ and since equality must hold throughout, $\left\|K_{s}\right\|=1$ and $x$ and $K_{s}$ are linearly dependent: $x= \pm K_{s}$. Accordingly the set $(K) \cup(-K)$ contains the surface of the unit sphere

Presented to the Society, December 27, 1954; received by the editors February 8 , 1956.

1 Work performed under contract N58304 with Office of Naval Research. 
of $\mathfrak{F C}$. If $\mathfrak{H C}$ is infinite-dimensional then, since that surface is weakly dense in the unit sphere the weakly compact $(K) \cup(-K)$ is the unit sphere. It follows that $K$ is a closed second category set in $\mathcal{H C}$ and therefore that it contains an interior point. If $\mathfrak{H C}$ is finite-dimensional the projection of $(K) \cup(-K)$ on any proper subspace of $\mathcal{H C}$ is easily seen to coincide with the unit sphere of that subspace, from which it follows as above that the projection of $K$ contains a sphere in the subspace.

Whatever the dimension of $\mathcal{F}$ may be, then, if $P$ is the projection of $\mathcal{H}$ onto the subspace spanned by the $k$ elements $x_{i}, i=1,2, \cdots, k$ the set $P K$ contains a sphere in $P$ Fe. The functions $x_{i}(t)=\left(x_{i}, P K_{t}\right)$ are the coordinates relative to the basis elements $x_{i}$ of the continuous curve $X(t)=P K_{t}$ in $P \mathcal{F}$ which covers the set $P K$ and is therefore a space-filling curve.

For $N=2$ our theorem does not contain any real information, since any nonconstant function determines a space-filling curve in $R_{1}$. Thus the subspace of $C(0,1)$ spanned by $x(t)=\cos (\pi t)$ and $y(t)=\sin (\pi t)$ is isometric to the Euclidean plane; for higher dimensions, however, no such regular functions can occur.

Professor K. T. Smith has pointed out to the author that the foregoing theorem holds not only for Hilbert space, but for any real Banach space $E$ with strictly convex dual. For if $K$ denotes the set of evaluation functionals regarded as functionals on $E$, then $(K) \cup(-K)$ is a weak-star compact subset of $E^{*}$ the weak-star closed, convex hull of which coincides with the unit sphere of $E^{*}$. Thus $(K) \cup(-K)$ contains all the extreme points of that unit sphere, i.e. all functionals of norm 1. The argument then proceeds as in the case of Hilbert space. Since any separable Banach space can be normed without changing the topology in such a way that the dual space becomes strictly convex, it follows that the situation described in the theorem depends only on the metric of the space and not on the topology.

\section{REFERENCES}

1. N. Aronszajn, Theory of reproducing kernels, Trans. Amer. Math. Soc. vol. 68 (1950) pp. 337-404.

2. S. Banach, Théorie des opérations linéaires, Warsaw, 1932.

UNIVERSITY OF KANSAS 